\title{
Ethanolic Extract of Nigella sativa Seeds Lacks the Chemopreventive Efficacy in the Post Initiation Phase of DMH-Induced Colon Cancer in a Rat Model
}

\author{
Wasfi Asfour $^{1 *}$, Sawsan Almadi ${ }^{1}$, Lina Haffar ${ }^{2}$ \\ ${ }^{1}$ Department of Pharmacology and Toxicology, College of Pharmacy, Damascus University, Damascus, Syria; ${ }^{2}$ Department of Histo- \\ pathology, College of Medicine, Damascus University, Damascus, Syria. \\ Email: "asfour_wasfi@yahoo.com
}

Received January $22^{\text {nd }}, 2013$; revised February $28^{\text {th }}, 2013$; accepted April $6^{\text {th }}, 2013$

Copyright (C) 2013 Wasfi Asfour et al. This is an open access article distributed under the Creative Commons Attribution License, which permits unrestricted use, distribution, and reproduction in any medium, provided the original work is properly cited.

\begin{abstract}
Nigella sativa, belonging to the Ranunculacea family, is a versatile phytochemical mine bestowed with multi-dimensional medicinal effects. The current study was performed to investigate the chemopreventive efficacy, if any, of the ethanolic extract of indigenous Nigella sativa seeds (ENS) and to evaluate its potentials on tumor progression during the initiation and post initiation phases of Dimethylhydrazine-induced colon carcinogenesis in a rat model. ENS treatment during the initiation phase unveiled chemopreventive effect manifested by significant reductions in tumor incidence, multiplicity and tumor volumes. Histopathological findings and modified Duke's classification of tumors provided evidence that ENS, administered in the initiation phase, is capable of delaying progression, restricting invasion and attenuating aggressiveness of colon tumors. These results imply that ENS may be a promising candidate in the primary prevention of colon cancer. On the contrary, we demonstrated that ENS lacked chemopreventive and tumorigenesis inhibitory effects in the post initiation phase. We speculate that the chemopreventive effect of ENS might be due to the synergistic actions of various constituents present in the extract. However, extensive studies are warranted and more efforts need to be dedicated to fractionate, analyze and to further appraise the anticancer effect of ENS before any definitive conclusions can be drawn.
\end{abstract}

Keywords: Lack; Ethanolic Extract; Nigella sativa; Dimethylhydrazine; Post Initiation; Colon Cancer; Rat; Modified Duke's Classification

\section{Introduction}

Cancer is a disease with a major worldwide burden, and it is now the second leading cause of mortality in western developed countries preceded only by cardiovascular diseases [1].

Colorectal cancer is the third most frequently diagnosed malignancy worldwide and one of the leading causes of cancer-related mortality [2].

In Syria the incidence and mortality rates of colorectal cancer are increasing at an alarming rate in recent decade, being the second most prevalent type of cancer (second to lung \& bronchus cancer in men, and breast cancer in women). It accounts for $12 \%$ of cancer incidence in males and females [3].

Despite the tremendous efforts that have been devoted

\footnotetext{
"Corresponding author.
}

to the development of treatment strategies, therapy modalities alone have not been fully effective in countering either the high incidence or low survival rate of colorectal cancer. The failure to control deaths from colorectal cancer provided the rational to develop strategies for prevention. The development of chemopreventive agents to prevent intraepithelial neoplasia such as benign adenomas and early neoplastic lesions represents a significant advance in clinical cancer prevention research [4].

Since immemorial times, it has been relied on herbs for the successful prevention and cure of a broad array of maladies.

In recent era, the versatile chemopreventive and therapeutic potentials of medicinal herbs attracted the widespread attention of cancer prevention and treatment researchers to investigate and to harness these potentials in the struggle against carcinogenesis. Consequently, phy- 
tochemical compounds burgeoned as a new generation of chemotherapeutic (paclitaxel, vincristine, etc.) and chemopreventive agents. In this regard, Nigella sativa is a prominent candidate that warrants further investigation as a cancer chemopreventive agent.

Nigella sativa, first identified and described by Linnaeus in 1753 [5], is an annual herbaceous plant belonging to the Ranunculacea family, native to southwest Asia [6]. It is especially grown in the East Mediterranean countries for its "Black seeds". Seeds have been employed as a spice and food preservative in eastern civilization [7]. Ample and consistent experimental studies provided conclusive evidence that Nigella sativa seeds are bestowed with natural medicinal potentials. In fact, literature is replete with the subject of pharmacological actions of a single component or an extract of Nigella sativa seeds [8]. These multi-dimensional, "nutraceutical" actions include analgesic [9] antimicrobial [10,11], antidiabetic [12], anti-hypertensive [13,14], antihistaminic $[15,16]$, immunopotentiating [17,18], anti-inflammatory [19-21], antioxidant and radical scavenging actions [2224]. The antineoplastic effects of $N$. sativa seed and its extracts were investigated both in vivo using animal models and in vitro using cancer cell lines [25]. An antitumor activity of $N$. sativa seeds has been reported by Salomi et al. [26] who showed that a crude methanol extract of black seed exhibited a strong cytotoxic action on Erlich ascites carcinoma, Dalton's ascites lymphoma and sarcoma 180 cells. They also demonstrated that extracts of the seeds inhibited the growth of Erlich ascites carcinoma in mice [26]. In addition, topical application of $N$. sativa seeds extract inhibited skin dimethylbenz(a)anthracene induced carcinogenesis in mice, delayed the onset of papilloma formation and reduced the number of papillomas per mouse [27]. In the same study, intraperitoneal administration of $100 \mathrm{mg} / \mathrm{kg}$ of $N$. sativa extract restricted soft tissue sarcomas induced in albino mice by 20 -methylcholanthrene to $33 \%$ [27]. Another study [28] evaluated in vitro a crude gum, a fixed oil and two purified components of the seed (Thymoquinone: TQ and dithymoquinone: DTQ) for cytotoxicity to some parental and multidrug-resistant human tumor cell lines. The results indicated that the gum and the oil were devoid of cytotoxicity, while both TQ and DTQ were cytotoxic to all cell lines tested. Recent in vitro studies reported the ability of TQ to inhibit the proliferation of colon cancer cells $[29,30]$, lymphoblastic leukemia cells [31], laryngeal carcinoma cells $[32,33]$, and prostate cancer cells $[33,34]$ and so on. Furthermore, $\alpha$-hederin which is a triterpene saponin isolated from $N$. sativa showed a significant tumor inhibitory effect in mice [35].

The core purpose of this in vivo research was to investigate the chemopreventive efficacy, if any, of the etha- nolic extract of indigenous Nigella sativa seeds (ENS) against DMH-induced carcinogenesis in a rat model and to appraise its potential impact on tumor progression and invasion through a two-phase study: an initiation phase and a post-initiation phase.

\section{Materials and Methods}

\subsection{Materials}

\section{Animals and Chemicals}

For the purpose of the study eighty male albino wistar rats aged 5 weeks were obtained from LEEN Company for laboratory animals (Damascus, Syria). Animals were kept in the animal care facility (college of Pharmacy, Damascus University). They were housed four per polypropylene cage with a wire-mesh top and a hygienic bed of sawdust in a specific pathogen-free animal room and maintained in a conventional controlled environment $\left(24^{\circ} \mathrm{C} \pm 1{ }^{\circ} \mathrm{C}, 50 \% \pm 10 \%\right.$ relative humidity with an alter-

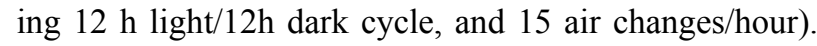
Rats had unrestricted access to a basal commercial rodents pellet diet and tap water ad libitum.

All the procedures were performed in accordance with the standard guidelines for care and use of laboratory animals and the protocols followed were approved by the local University Ethics Committee for Animal Research.

Indigenous dried seeds of Nigell sativa L. were procured from the local market and their authenticity was confirmed. 1,2-Dimethylhydrazine dihydrochloride (DMH) was purchased from Acros Organics (Morris Plains, NJ, USA). Thymoquinone (TQ) standard (99\%) was obtained from Sigma-Aldrich (St. Louis, MO, USA). Reagents (HPLC grade) were purchased from Merck (Darmstadt, Germany).

\subsection{Methods}

\subsubsection{Preparation of Nigella sativa Seeds Extract}

Seeds were washed, dried, and crushed into coarse powder with an electric mill. The powder was exhaustively extracted with $90 \%$ ethanol at room temperature. The extract was filtered and the vehicle was evaporated under vacuum.

\subsubsection{Analysis of Thymoquinone Content in the Administered Dose of ENS}

Thymoquinone content was determined using an isocratic normal phase HPLC method described by Aboul-Enein, H. and Abou-Basha, L. [36].

\subsubsection{Preparation of Carcinogen}

1,2-Dimethylhydrazine dihydrochloride (DMH) was dissolved in $1 \mathrm{mMol} / \mathrm{L}$ EDTA-normal saline; the $\mathrm{pH}$ was 
adjusted to 6.5 with $1 \mathrm{Mol} / \mathrm{L} \mathrm{NaOH}$ to ensure the $\mathrm{pH}$ suitability and the stability of the chemical. The resultant solution of the carcinogen was used immediately after preparation.

A full-face respirator equipped with a combination filter cartridge (The Advantage ${ }^{\circledR}$ 1000: MSA Co., USA) was used during the preparation of the carcinogen solution and when injecting animals.

DMH was injected subcutaneously (s.c.) at a dose of $40 \mathrm{mg} / \mathrm{kg} /$ body weight once a week for ten consecutive weeks on the dorsal back of the animal.

\subsubsection{Experimental Design}

Before the initiation of the experiment, animals were adapted to the conditions they were kept in for a week. They were then randomly split up into five groups:

1) Normal control group $(n=16)$ : Rats in this group served as negative controls. They were also injected subcutaneously, at weekly intervals for 10 weeks, with 0.5 $\mathrm{ml}$ of $1 \mathrm{mMol} / \mathrm{L}$ EDTA-saline which was used as a vehicle for the $(\mathrm{DMH})$ treatments.

2) DMH group $(n=16+8)$ : served as carcinogen control or positive control. Rats in this group were given subcutaneous injections of (DMH) once a week at a dose of $40 \mathrm{mg} / \mathrm{kg}$ body weight for 10 consecutive weeks. By the end of the initiation phase, i.e. at the beginning of the 11 th week, 8 animals of this group were sacrificed in order to identify the histological alterations in the colonic histoarchitecture at this point of the carcinogenesis process.

3) (DMH + ENS initiation) group $(n=16)$ : received the same DMH treatment as in group II and simultaneously gavaged ENS at a daily dose equivalent to $150 \mathrm{mg}$ of the powdered seeds $/ \mathrm{kg}$ body weight for the whole 10 weeks period of the initiation phase.

4) $($ DMH + ENS post-initiation) group $(n=16)$ : Animals were subjected to the same carcinogen protocol as in group II and subsequently gavaged ENS at a daily dose equivalent to $150 \mathrm{mg}$ of the powdered seeds $/ \mathrm{kg} /$ body weight during the last 11 post-initiation weeks of the experimental period.
5) And Animals in (ENS group) $(n=8)$ were intubated ENS alone at a daily dose equivalent to $150 \mathrm{mg}$ of the powdered seeds $/ \mathrm{kg} /$ body weight for the whole period of the experiment ( 22 weeks).

Table 1 summarizes the design of the experiment.

\subsubsection{Body Weights Record}

A record of the body weights of normal control, DMH and ENS-treated animals was kept throughout the study. The animals were weighed at the beginning of the experiment, once a week during the experiment and finally before sacrifice.

\subsubsection{Macroscopical Analysis of Induced Tumors}

By the end of the 22nd week, rats were sacrificed. They were then autopsied, and the colons were excised, slit open along the longitudinal axis, flushed with saline, and the inner surface was examined macroscopically. The number of tumors in each colon was counted. The length $(\mathrm{L})$, width $(\mathrm{W})$ and height $(\mathrm{H})$ of tumor were measured using a vernier caliper with $0.1 \mathrm{~mm}$ graduations, and Tumor Volume (TV) was calculated $(\mathrm{TV}=\mathrm{L} \times \mathrm{W} \times \mathrm{H} \times$ $\pi / 6)$.

The chemopreventive tumor response was assessed on the basis of tumor incidence (percentage of animals having tumors), multiplicity (mean of tumors counted/animals) and mean tumor volume (mean size of tumors in tumor-bearing rats.

\subsubsection{Tissue Processing}

After the gross pathologic changes (number, and dimensions) were recorded, the colons were fixed flat between pieces of filter paper in $10 \%$ phosphate-buffered formalin for $24 \mathrm{~h}$.

Afterwards, tissue samples were dehydrated through ascending grades of alcohol, cleared in xylene and embedded in paraffin. $5-\mu \mathrm{m}$ thick sections were cut and stained with routine hematoxylin and eosin (H\&E) for histological diagnosis of tumors. Major organs and lymph nodes were also excised, fixed in $10 \%$ phosphate-buffered formalin and then prepared for histological analysis.

Table 1. Experimental design.

\begin{tabular}{|c|c|c|}
\hline Experimental groups & $\begin{array}{l}\text { Number of rats } \\
\text { (n) }\end{array}$ & Protocol \\
\hline Normal control & $(16)$ & Subcutaneous (s.c.) injections of mMol/L EDTA-saline (vehicle of DMH) for 10 consecutive wk. \\
\hline DMH & $(16+8)$ & $\begin{array}{l}\text { Weekly (s.c.) injections of (DMH) at a dose of }(40 \mathrm{mg} / \mathrm{kg}) \text { for } 10 \text { consecutive wk. } \\
\text { (Eight rats were sacrificed at the end of the } 10 \text { th } \mathrm{wk}) \text {. }\end{array}$ \\
\hline DMH + ENS initiation & $(16)$ & $\begin{array}{l}\text { Same DMH treatment }+ \text { simultaneously treated with a daily dose of ENS (equivalent to } 150 \mathrm{mg} \\
\text { of the powdered seeds } / \mathrm{kg} \text { body weight) for } 10 \mathrm{wk} .\end{array}$ \\
\hline DMH + ENS post-initiation & $(16)$ & $\begin{array}{l}\text { Same DMH treatment, subsequently treated with a daily dose of ENS (equivalent to } 150 \mathrm{mg} \text { of } \\
\text { the powdered seeds } / \mathrm{kg} \text { body weight) from the } 11 \text { th to the } 22 \mathrm{nd} \mathrm{wk} \text {. }\end{array}$ \\
\hline ENS & (8) & Daily dose of ENS (equivalent to $150 \mathrm{mg}$ of the powdered seeds $/ \mathrm{kg}$ ) for $22 \mathrm{wk}$. \\
\hline
\end{tabular}


Colons of the eight rats from DMH group, sacrificed at the end of the 10th week, were prepared at the time and stained with (H\&E) for histopathological observations at the light microscopic level.

\subsubsection{Statistical Analysis}

All Statistical analyses were performed using the (GraphPad Prism5) statistical software. Differences in mean body weight, multiplicity and tumor volume in the different groups were determined using one-way analysis of variance (ANOVA) followed by Tukey's Multiple Comparison test, while the significance of differences in tumor incidence between experimental groups was assessed by Fisher's exact test. All tests were performed two-sided.

Differences in histological grades of colon tumors among the groups were analyzed by Kruskal-Wallis test followed by Dunn's Multiple Comparison test. Statistical significance was accepted when $P<0.05$.

\section{Results}

\subsection{Thymoquinone Content in the Administered Dose of ENS}

HPLC analysis revealed that the content of thymoquinone equals $0.026 \mathrm{mg}$ in the employed dose of ENS in this study.

\subsection{General Observation and Body Weight Changes}

No apparent signs of severe toxicity were observed in animals received ENS. All rats survived until the final termination and maintained a relatively healthy appearance throughout the experiment.

By the end of 22nd week, final average body weight of the rats in (DMH) group was remarkably decreased compared with the normal control group $(P<0.05)$. Body weights of rats in (DMH + ENS initiation) group were decreased; however, the reductions were not statistically significant when compared to the normal control group. On the contrary, average body weight of (DMH + ENS post-initiation) group was significantly reduced in comparison with the normal group.

The variations in body weights of the animals subjected to different treatments are shown in Figure 1.

Histopathological analysis of colons of rats $(n=8)$ from DMH group, sacrificed at the end of the 10th week (end of the initiation phase), revealed colonic hyperplasia at that point of the experiment, which was the starting point for the post-initiation phase.

\subsection{Tumor Incidence}

Macroscopically, the distribution of colon tumors was

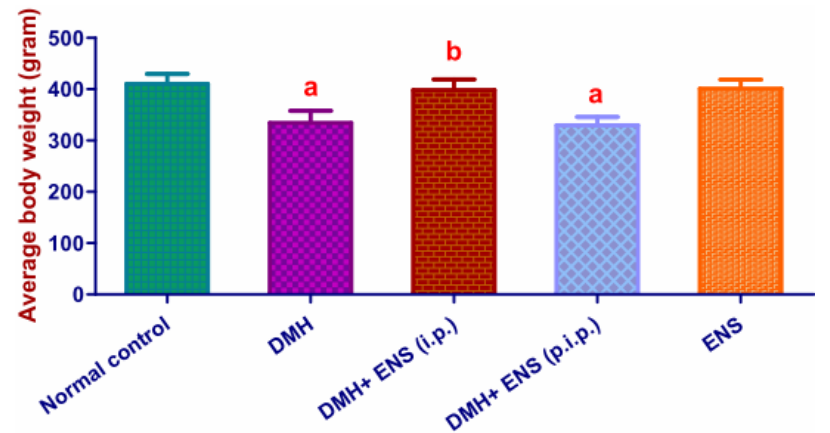

(i.p.): initiation phase; (p.i.p.): post initiation phase; Values are means $\pm \mathrm{SD}$. asignificantly decreased $(P<0.05)$ by one-way ANOVA followed by Tukey's Multiple Comparison test, when values were compared to the normal control group; 'significantly higher $(P<0.05)$ by one-way ANOVA followed by Tukey's Multiple Comparison test, when values were compared to $\mathrm{DMH}$ group.

Figure 1. Final average body weights.

predominantly observed in the distal and middle colon at the end of 22nd week. All rats in DMH group developed colon cancer at the end of this study whereas, neither the animals of normal control group nor those of ENS (alone) group developed colon tumors.

Table 2 summarizes the incidences of DMH-induced colon tumors in the DMH group and ENS-treated groups.

Two rats in (DMH) group and two rats in (DMH + ENS post-initiation) group developed an ear tumor each. Histopathalogical analysis of the tumors revealed Squamous Papilloma.

\subsection{Tumor Multiplicity}

ENS decreased the mean number of colon tumors in the initiation phase. However, ENS treatment in the post initiation phase slightly and insignificantly reduced multiplicity. Data are shown in Table 3.

\subsection{Tumor Volume}

Mean tumor volume was significantly reduced in consequence to ENS treatment in the initiation phase, whereas ENS administered during the post initiation phase failed to exert remarkable effect on the sizes of tumors. Table 4 displays these results.

\subsection{Histopathological Evaluation and Tumors Grading}

Animals with DMH-induced colon cancer characteristically develop multiple tumors and each tumor is at a different histological stage [37].

As Peto [38] has pointed out the heterogeneity of histological stages of malignancy within each animal makes it impossible to accurately classify the extent of the disease in each animal as a whole. Comparisons of total tumor numbers between whole animals, when each tu- 
Table 2. Efficacy of orally administered ENS $(150 \mathrm{mg} / \mathrm{kg} /$ day) on colonic tumor incidence.

\begin{tabular}{ccc}
\hline \multirow{2}{*}{ Group } & \multicolumn{2}{c}{ Incidence } \\
\cline { 2 - 3 } & No. & $(\%)$ \\
\hline Normal control & 0 & $(0)$ \\
DMH & 16 & $(100)$ \\
DMH + ENS (i.p.) & 10 & $(62.5)^{\mathrm{a}}$ \\
DMH + ENS (p.i.p.) & 15 & $(93.75)$ \\
ENS & 0 & $(0)$ \\
\hline
\end{tabular}

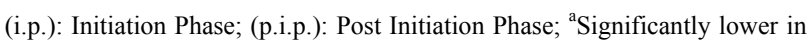
comparison with DMH group using Fisher's exact test $(P<0.05)$.

Table 3. Efficacy of orally administered ENS $(150 \mathrm{mg} / \mathrm{kg} /$ day) on tumor multiplicity.

\begin{tabular}{cc}
\hline Group & Multiplicity \\
\hline Normal control & 0 \\
DMH & $5.37 \pm 2.19$ \\
DMH + ENS (i.p.) & $3.06 \pm 1.32^{\mathrm{a}}$ \\
DMH + ENS (p.i.p.) & $4.3 \pm 1.91$ \\
ENS & 0 \\
\hline
\end{tabular}

(i.p.): Initiation Phase; (p.i.p.): Post Initiation Phase. Data are expressed as mean \pm S.D; ${ }^{\text {a }}$ Significantly lower in comparison with DMH group using one-way-ANOVA followed by Tukey's Multiple Comparison test, $P<0.05$.

Table 4. Efficacy of orally administered ENS $(150 \mathrm{mg} / \mathrm{kg} /$ day) on tumor volume.

\begin{tabular}{cc}
\hline Group & Tumor volume \\
\hline Normal control & - \\
DMH & $518.1 \pm 61.13$ \\
DMH + ENS (i.p.) & $256.47 \pm 49.08^{\mathrm{a}}$ \\
DMH + ENS (p.i.p.) & $478.23 \pm 63.72$ \\
ENS & - \\
\hline
\end{tabular}

(i.p.): Initiation Phase; (p.i.p.): Post Initiation Phase. Data are expressed as mean \pm S.D; ${ }^{\text {a }}$ Significantly lower in comparison with DMH group using one-way-ANOVA followed by Tukey's Multiple Comparison test, $P<0.05$.

mor within each animal is at a different histological stage, are thus of doubtful validity. Consequently, for the purpose of this experiment, animals were staged with reference to a single index tumor, defined as the largest macroscopically identifiable colon tumor.

Colon tumors were divided into adenoma and carcinoma. Grades of carcinoma were allocated according to modified Duke's pathological classification [39].

Histopathological findings are summarized in Table 5.

Four cases of mucinous adenocarcinoma (signet-ring adenocarcinoma) were observed in (DMH) group, and four cases in (DMH + ENS post-initiation) group. Although mucinous adenocarcinoma is considered a more aggressive subtype of adenocarcinoma associated with particularly poor prognosis, all mucinous adenocarcinoma cases in the present study were classified as carci-
Table 5. Numbers of rats with DMH-induced colon tumors allocated to one of six histological grades.

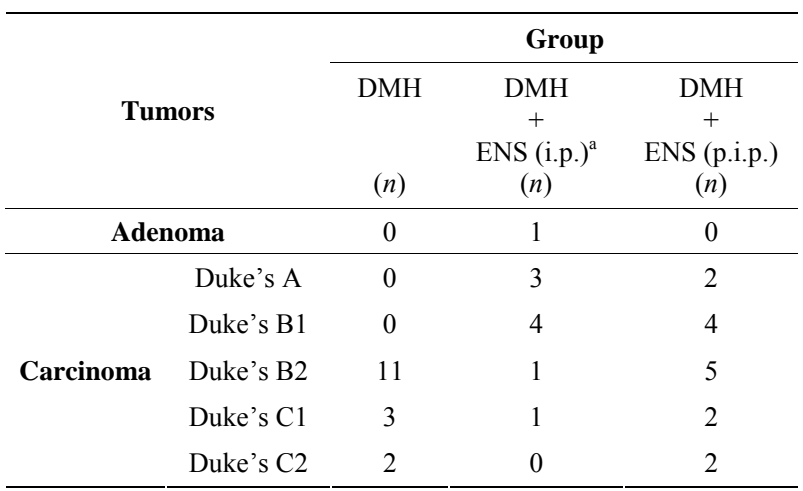

$\mathrm{n}$ : Number of Rats in each histological grade. ${ }^{\mathrm{a}} \mathrm{Histological}$ grade of $(\mathrm{DMH}$ + ENS initiation) group is less progressive and less invasive when compared with DMH (carcinogen control) group. Statistical significance was assessed by Kruskal-Wallis test followed by Dunn's Multiple Comparison test.

noma and graded according to modified Duke's staging.

\section{Discussion}

The antitumor effects of $N$. sativa was recognized by Avicenna [40] who generally used $N$. sativa for the treatment of tumors, particularly hard splenic mass.

With regard to modern science a few experiments were performed, concerning the anticancer effect, using the ethanolic extract of the seeds both for in vivo and in vitro studies [8].

An (in vitro) study demonstrated that ethylacetate column chromatographic fraction (CC-5) of an ethanolic extract of $N$. sativa exhibited cytotoxic effects against different types of cancer cell lines, such as, P388, HepG2, Molt4 and Lewis lung carcinoma cells [41].

The present in vivo study investigated the chemopreventive efficacy of the ethanolic extract of indigenous Nigella sativa seeds against DMH-induced colon carcinogenesis in a rat model during the initiation and post initiation phases.

The results demonstrated that ENS, at a dose equivalent to $150 \mathrm{mg}$ powdered seed $/ \mathrm{kg}$ body weight, has an inhibitory effect against chemically induced colon carcinogenesis when administered in the initiation phase. This effect was manifested by a significant decrease in tumor incidence of the order of $38 \%$, an appreciable reduction in multiplicity by $43 \%$ and a remarkable decline in tumor volume by $50 \%$.

However, on the contrary, although ENS treatment in the post-initiation phase tended to decrease incidence, multiplicity and tumor sizes, these reductions were not significant statistically, implying that ENS exerted no carcinogenesis suppressive effect during the post-initiation phase.

A possible explanation of these different effects of 
ENS in the initiation phase and during the post-initiation phase may be suggested to be the result of the radical scavenging and antioxidant activities of ENS which, as a consequence, led to anti-toxic effects against insults that might have been induced by the carcinogen, and/or the enhancement of detoxification processes of the carcinogen during the initiation phase.

It seems reasonable that ENS might have an antimutagenic activity and/or a recovery potential at the chromosomal level in the initiation phase but lacks the suitable cytotoxic potency required to combat colonic transformed cells and to adequately restrict tumorigenesis process and thereby to obstruct the progression of colonic hyperplastic pre-malignant lesions, which arose in the colonic histoarchitecture at the beginning of the post initiation phase, into frank invasive tumors.

In other words, ENS possibly targets more effectively the earliest stages in DMH-induced colon carcinogenesis.

In addition, the severity of DMH protocol used to induce colon carcinogenesis is also crucial and it is a major determinant of the consequences of tumorigenesis process in the post initiation phase. It might be that ENS has somewhat of a cytotoxic effect, but the intensity of our protocol exceeded the capacity of ENS, at the employed dose level, for completely counteracting the profound molecular and histological changes induced by the carcinogen and therefore, ENS failed to exert a chemopreventive effect in the post-initiation phase.

The protocol followed herein to induce colon cancer (40 $\mathrm{mg}$ of $\mathrm{DMH} / \mathrm{kg}$ body weight/once a week for ten consecutive weeks) is obviously more vigorous when compared, for example, to the protocol followed in the study of Salim and Fukushima [42] through which they demonstrated that the daily treatment with $N$. sativa volatile oil has the ability to inhibit 1,2-dimethylhydrazine induced colon carcinogenesis of rats in the postinitiation stage as reflected by significant reductions in aberrant crypt foci (ACF), the putative preneoplastic lesions for colon cancer.

Since previous studies attributed the efficacy of Nigella sativa seeds to thymoquinone (TQ) and employed [43-45] relatively large doses $(6$ and $10 \mathrm{mg} / \mathrm{kg}$ body weight) of TQ to establish remarkable anticancer effects, the inadequate meager administered dose of TQ $(0.026 \mathrm{mg} / \mathrm{kg})$, presented in ENS used in this work, during the post initiation phase might be an important factor and one of the causes underlying the insignificant impact of ENS on colon carcinogenesis and the lack of anticancer effect in the post initiation phase. However, since TQ is a liposoluble benzoquinone [46] and its solubility is relatively low in water [47], substantially meager concentration is expected in aqueous extracts of Nigella sativa seeds. Nonetheless, the aqueous extract of $N$. sativa was proven to have an anticancer effect. Farah and Begum [48] indicated that aqueous and alcohol extracts of $N$. sativa were found to be effective (in vitro) in inactivating MCF-7 breast cancer cells. It has also been shown that $N$. sativa, in combination with melatonin and retinoic acid reduced the carcinogenic effects of DMBA (7,12-dimethylbenz(a)anthracene) in mammary carcinoma of rats [49].

Furthermore, another study [50] carried out on standardized aqueous and ethanolic extracts of a plant mixture (Nigella sativa, Hemidesmus indicus, and Smilax glabra) determined their cytotoxic effects on human hepatoma (HepG2) cell lines. The study demonstrated that both extracts exhibited strong dose dependent cytotoxicity to HepG2 cells. The aqueous extract showed a significantly higher cytotoxic potential than the ethanolic extract.

Accordingly, the participation of various constituents other than thymoquinone, with their different mechanisms of actions, and the various possible synergistic effects of such constituents can not be excluded as a source of the chemopreventive and anticancer effects of ENS. TQ is not necessarily the only compound to which the chemopreventive and anticancer actions of Nigella sativa seeds could be attributed. A previous study has even demonstrated that TQ had no effect on HT-29 (colon adenocarcinoma) cell line [51].

Taking the aforementioned into account and since the composition of Nigella sativa, like that of most herbs, varies with the geographic distribution, time of harvest and horticultural practices, it is advisable that the ENS will have to be fractionated, analyzed and further investigated in order to identify the responsible compounds for the chemopreventive action, with regard to colon carcinogenesis, and to elucidate the mechanism(s) underlying its possible effects.

Tumor formation was adopted as an end point of this experiment. The staging system employed, which is analogous to the modified Duke's staging system for human colorectal cancer, provides accurate prognostic information. In other words, cases with less advanced tumors (stage A) can survive significantly longer than those with more advanced tumors (stage B and C), irrespective of treatment.

Histopathological analysis of tumors and the histological grading of carcinoma, using modified Duke's classification, indicated that rats in ENS-treated group during the initiation phase tended to develop considerably less progressive tumors and that the extent to which carcinomas invade through the histological layers of the colon was remarkably reduced.

To our knowledge, this (in vivo) study is the first work in a rat model of DMH-induced colon cancer which pro- 
vided evidence that ENS treatment in the initiation phase effectively delayed and hindered tumorigenesis process as reflected by restricted tumor progression and declined extent of invasion. Two animals of DMH group developed carcinomas classified as Duke's stage C2 implying increased invasion (tumor penetrates through muscularis propria), lymph node involvement and poorer prognosis, while none of the animals of ENS-treated group in the initiation phase had such an advanced stage, suggesting improved prognosis and less progression and invasion.

Unfortunately, ENS treatment during the post initiation phase had no effect on the progression of colonic hyperplastic foci. From the histopathological point of view, our results indicated that invasive and progressive colonic tumors, which do not differ significantly from those of DMH group, were formed in (DMH + ENS postinitiation) group.

It is worth mentioning that, although mucinous adenocarcinoma cases in the present work were classified as carcinomas, the differences encountered among the experimental groups regarding the incidence of such more aggressive type of carcinoma must be taken into consideration when evaluating the protective effect of ENS against induced colon cancer in vivo.

In view of our results, concerning the incidence of mucinous adenocarcinoma, we content that ENS has the potential to attenuate the aggressiveness of colon carcinogenesis when administered during the initiation phase.

The overall histological findings clearly indicate and expand the database that ENS might play a promising inhibitory role with respect to colon carcinogenesis when administered during the initiation phase. Moreover, the absence of tumor incidence in rats treated with ENS alone for a long period ( $22 \mathrm{wk}$ ) suggests ENS, at this dose level, causes no disruption of normal cellular homeostasis and hence is non-toxic.

\section{Conclusions}

In summary, the salient finding to emerge from this study is that the ethanolic extract of indigenous Nigella sativa seeds (ENS), when administered during the initiation phase at a dose equivalent to $150 \mathrm{mg}$ of powdered seeds/ $\mathrm{kg}$ body weight, is bestowed with a chemopreventive efficacy against DMH-induced colon carcinogenesis despite its relatively low content of TQ. We provided evidence that ENS is capable of delaying progression, restricting invasion and attenuating aggressiveness of colon tumors in a rat model.

On the contrary, ENS was demonstrated to be devoid of chemopreventive and tumorigenesis inhibitory effects when administered during the post initiation phase of chemically induced colon cancer.
We speculate that the chemopreventive effect of ENS during the initiation phase might be due to the synergistic actions of various constituents present in the extract. Further detailed studies are warranted on ENS to confirm herein proposed hypotheses, regarding the lack of anticancer effect of ENS in the post initiation phase, before any definitive conclusions can be drawn.

\section{REFERENCES}

[1] J. Ferlay, F. Bray, P. Pisani and D. Parkin, "GLOBOCAN 2002: Cancer Incidence, Mortality and Prevalence Worldwide," IARC Cancer Base, Vol. 5, IARC Press, Lyon, 2004.

[2] J. Ferlay, H. R. Shin, F. Bray, D. Forman, C. Mathers and D. M. Parkin, "GLOBOCAN 2008 v1.2, Cancer Incidence and Mortality Worldwide," IARC Cancer Base, Vol. 10, International Agency for Research on Cancer Press, Lyon, 2010. http://globocan.iarc.fr

[3] Z. Msallati, A. El Jord and F. Al-Jerf, "Cancer Incidence in Syria, National," Cancer Registry, Cancer Prevention Directorate, Ministry of Health, Damascus, 2009.

[4] E. T. Hawk, A. Umar, J. L. Viner, "Colorectal Cancer Chemoprevention: An Overview," Gastroenterology, Vol. 126, No. 5, 2004, pp. 1423-1447.

doi:10.1053/j.gastro.2004.03.002

[5] P. C. M. Jansen, "Spices, Condiments and Medical Plants in Ethiopia: Their Taxonomy and Agricultural Significance," Center for Agricultural Publishing and Documentation, Addis Ababa, 1981, pp. 76-85.

[6] S. I. Saad, "Classification of Flowering Plants," 2nd Edition, The General Egyptian Book Co., Alexandria, 1975, pp. 412-413.

[7] M. F. Ramadan, "Nutritional Value, Functional Properties and Nutraceuticals Applications of Black Cumin (Nigella sativa L.): An Overview," International Journal of Food Science \& Technology, Vol. 42, No. 10, 2007, pp. 12081218. doi:10.1111/j.1365-2621.2006.01417.x

[8] K. E. El Tahir and D. Bakeet, "The Black Seed Nigella sativa Linnaeus-A Mine for Multi Cures: A Plea for Urgent Clinical Evaluation of Its Volatile Oil," Review, Journal of Taibah University for Medical Sciences, Vol. 1, No. 1, 2006, pp. 1-19.

[9] M. Abdel-Fattah, K. Matsumoto and H. Watanabe, "AntiNociceptive Effects of Nigella sativa Oil and Its Major Components in Mice," European Journal of Pharmacology, Vol. 400, No. 1, 2000, pp. 89-97. doi:10.1016/S0014-2999(00)00340-X

[10] T. El-Alfy, H. El-Fatatry and Toama, "Isolation and Structure Assignment of an Anti-Microbial Principle from the Volatile Oil Nigella sativa L.," Pharmazia, Vol. 30, 1975, pp. 109-111.

[11] M. N. Morsi, “Antimicrobial Effect of Crude Extracts of Nigella sativa on Multiple Antibiotics-Resistant Bacteria," Acta Microbiologica Polonica, Vol. 49, No. 1, 2000, pp. 63-74. 
[12] A. Al-Hader, M. Aqel and Z. Hassan, "Hypoglycaemic Effects of the Volatile Oil of Nigella sativa," Pharmaceutical Biology, Vol. 31, No. 2, 1993, pp. 96-100. doi:10.3109/13880209309082925

[13] K. E. El Tahir, M. M. Ashour and M. M. Al-Harbi, "The Cardiovascular Actions of the Volatile Oil of the Black Seed (Nigella sativa) in Rats: Elucidation of the Mechanism of Action," General Pharmacology: The Vascular System, Vol. 24, No. 5, 1993, pp. 1123-1131. doi:10.1016/0306-3623(93)90359-6

[14] F. R. Dehkordi and A. F. Kamkhah, "Antihypertensive Effect of Nigella sativa Seed Extract in Patients with Mild Hypertension," Fundamental \& Clinical Pharmacology, Vol. 22, No. 4, 2008, pp. 447-452. doi:10.1111/j.1472-8206.2008.00607.x

[15] M. Mahfouz, R. Abdel-Maguid and M. El-Dakhakhny, "The Effect of Nigellon Therapy on the Histaminopexic Power of Blood Sera Asthmatic Patients," Arzneimittelforschung, Vol. 15, 1965, pp. 1230-1231.

[16] M. H. Boskabady, N. Mohsenpoor and L. Takaloo, "Antiasthmatic Effect of Nigella sativa in Airways of Asthmatic Patients," Phytomedicine, Vol. 17, No. 10, 2010, pp. 707-713. doi:10.1016/i.phymed.2010.01.002

[17] R. Medinica, S. Mukerjee, T. Huschart and W. Corbitt, "Immunomodulatory and Anticancer Activity of Nigella sativa Plant Extract in Humans," Proceedings of the American Association for Cancer Research Annual Meeting, San Francisco, 10-13 April 1994, p. A2865.

[18] M. El Kadi, O. Kandil and A. M. Tabuni, "Nigella sativa and Cell Mediated Immunity," Archives of AIDS Research, Vol. 1, No. 5, 1990, pp. 232-235.

[19] P. J. Houghton, R. Zarka, B. De Las Heras and J. R. Hoult, "Fixed Oil of Nigella sativa and Derived Thymoquinone Inhibit Eicosanoid Generation in Leukocytes and Membrane Lipid Peroxidation," Planta Medica, Vol. 61, No. 1, 1995, pp. 33-36. doi:10.1055/s-2006-957994

[20] A. Mutabagani and S. A. El-Mahdy, "Study of the AntiInflammatory Activity of Nigella sativa L. and Thymoquinone in Rats," Saudi Pharmaceutical Journal, Vol. 5, No. 2-3, 1997, pp. 110-113.

[21] N. Chehl, G. Chipitsyna, Q. Gong, C. J. Yeo and H. A. Arafat, "Anti-Inflammatory Effects of the Nigella sativa Seed Extract, Thymoquinone, in Pancreatic Cancer Cells," HPB (Oxford), Vol. 11, No. 5, 2009, pp. 373-381. doi:10.1111/j.1477-2574.2009.00059.x

[22] S. M. Suboh, Y. Y. Bilto and T. A. Aburjai, "Protective Effects of Selected Medicinal Plants against Protein Degradation, Lipid Peroxidation and Deformability Loss of Oxidatively Stressed Human Erythrocytes," Phytotherapy Research, Vol. 18, No. 4, 2004, pp. 280-284. doi:10.1002/ptr.1380

[23] M. Burits and F. Bucar, "Antioxidant Activity of Nigella sativa Essential Oil," Phytotherapy Research, Vol. 14, No. 5, 2000, pp. 323-328. doi:10.1002/1099-1573(200008)14:5<323::AID-PTR621 3.0.CO;2-Q

[24] M. F. Ramadan, L. W. Kroh and J. T. Morsel, "Radical Scavenging Activity of Black Cumin (Nigella sativa L.),
Coriander (Coriandrum sativum L.), and Niger (Guizotia abyssinica Cass.) Crude Seed Oils and Oil Fractions," Journal of Agricultural and Food Chemistry, Vol. 51, No. 24, 2003, pp. 6961-6869. doi:10.1021/jf0346713

[25] M. A. Randhawa and M. S. Alghamdi, "A Review of the Pharmaco-Therapeutic Effects of Nigella sativa," Pakistan Journal of Medical Research, Vol. 41. No. 2, 2002, pp. 77-83.

[26] M. J. Salomi, S. C. Nair and K. R. Panikkar, "Inhibitory Effects of Nigella sativa and Saffron (Crocus sativus) on Chemical Carcinogenesis in Mice," Nutrition and Cancer, Vol. 16, No. 1, 1991, pp. 67-72. doi:10.1080/01635589109514142

[27] N. J. Salomi, S. C. Nair, K. K. Jayawardhanan, C. D. Varghese and K. R. Panikkar, "Antitumour Principles from Nigella sativa Seeds," Cancer Letters, Vol. 63, No. 1, 1992, pp. 41-46. doi:10.1016/0304-3835(92)90087-C

[28] D. R. Worthen, O. A. Ghosheh and P. A. Crooks, "The in Vitro Anti-Tumor Activity of Some Crude and Purified Components of Blackseed, Nigella sativa L.," Anticancer research, Vol. 18, No. 3, 1998, pp. 1527-1532.

[29] N. El-Najjar, M. Chatila and H. Moukadem, "Reactive Oxygen Species Mediate Thymoquinone-Induced Apoptosis and Activate ERK and JNK Signaling," Apoptosis, Vol. 15, No. 2, 2010, pp. 183-195. doi:10.1007/s10495-009-0421-z

[30] H. Gali-Muhtasib, D. Kuester and C. Mawrin, "Thymoquinone Triggers Inactivation of the Stress Response Pathway Sensor CHEK1 and Contributes to Apoptosis in Colorectal Cancer Cells," Cancer Research, Vol. 68, 2008, pp. 5609-5618. doi:10.1158/0008-5472.CAN-08-0884

[31] M. Alhosin, A. Abusnina and M. Achour, "Induction of Apoptosis by Thymoquinone in Lymphoblastic Leukemia Jurkat Cells Is Mediated by a p73-Dependent Pathway which Targets the Epigenetic Integrator UHRF1," Biochemical Pharmacology, Vol. 79, No. 9, 2010, pp. 12511260. doi:10.1016/j.bcp.2009.12.015

[32] K. Womack, M. Anderson and M. Tucci, "Evaluation of Bioflavonoids as Potential Chemotherapeutic Agents," Biomedical Sciences Instrumentation, Vol. 42, 2006, pp. 464-469.

[33] L. R. Richards, P. Jones and J. Hughes, "The Physiological Effect of Conventional Treatment with Epigallocatechin-3-gallate, Thymoquinone, and Tannic Acid on the LNCaP Cell Line," Biomedical Sciences Instrumentation, Vol. 42, 2006, pp. 357-362.

[34] L. R. Richards, P. Jones and H. Benghuzzi, "A Comparison of the Morphological Changes Associated with Conventional and Sustained Treatment with Pigallocatechin-3-gallate, Thymoquinone, and Tannic Acid on LNCaP Cells," Biomedical Sciences Instrumentation, Vol. 44, 2008, pp. 465-470.

[35] S. M. K. Swamy and T. K. H. Benny, "Extraction, Isolation and Characterization of Anti-Tumor Principle, Alpha-Hederin, from the Seeds of Nigella sativa," Planta Medica, Vol. 67, No. 1, 2001, pp. 29-32.

doi:10.1055/s-2001-10628 
[36] H. Aboul-Enein and L. Abou-Basha, "Simple HPLC Method for the Determination of Thymoquinone in Black Seed Oil (Nigella sativa Linn.)," Journal of Liquid Chromatography, Vol. 18, No. 5, 1995, pp. 895-902. doi:10.1080/10826079508010400

[37] K. M. Pozharisski, "Morphology and Morphogenesis of Experimental Epithelial Tumors of the Intestine," Journal of the National Cancer Institute, Vol. 54, No. 5, 1975, pp. 1115-1135.

[38] R. Peto, "Epidemiology, Multistage Models, and ShortTerm Mutagenicitytests," In: H. H. Hiatt, et al., Eds., Origins of Human Cancer, Cold Spring Harbour Laboratories, New York, 1977, pp. 1403-1438.

[39] V. B. Astler and F. A. Coller, "The Prognostic Significance of Direct Extension of Carcinoma of the Colon and Rectum," Annals of Surgery, Vol. 139, No. 6, 1954, pp. 846-852. doi:10.1097/00000658-195406000-00015

[40] M. A. Randhawa and M. S. Alghamdi, "Anticancer Activity of Nigella sativa (Black Seed)-A Review," The American Journal of Chinese Medicine, Vol. 39, No. 6, 2011, pp. 1075-1091. doi:10.1142/S0192415X1100941X

[41] S. M. Swamy and B. K. Tan, "Cytotoxic and Immunopotenciating Effects of Ethanolic Extract of Nigella sativa L. Seed," Journal of Ethnopharmacology, Vol. 70, No. 1, 2000, pp. 1-7. doi:10.1016/S0378-8741(98)00241-4

[42] E. I. Salim and S. Fukushima, "Chemopreventive Potential of Volatile Oil from Black Cumin (Nigella sativa L.) Seeds against Rat Colon Carcinogenesis," Nutrition and Cancer, Vol. 45, No. 2, 2003, pp. 195-202. doi:10.1207/S15327914NC4502 09

[43] S. Attoub, O. Sperandio, H. Raza and K. Arafat, "Thymoquinone as an Anticancer Agent: Evidence from Inhibition of Cancer Cells Viability and Invasion in Vitro and Tumor Growth in Vivo," Fundamental and Clininal Pharmacology, 2012 (online). doi:10.1111/j.1472-8206.2012.01056.X

[44] T. Yi, S. G. Cho, Z. Yi and X. Pang, "Thymoquinone Inhibits Tumor Angiogenesis and Tumor Growth through Suppressing AKT and ERK Signaling Pathways," Molecular Cancer Therapeutics, Vol. 7, No. 7, 2008, pp.

\section{Nomenclature}

ENS: Ethanolic extract of indigenous Nigella sativa Seeds

TQ: Thymoquinone

DTQ: Dithymoquinone

DMH: Dimethylhydrazine dihydrochloride
1789-1796. doi:10.1158/1535-7163.MCT-08-0124

[45] W. Asfour, S. Almadi and L. Haffar, "Thymoquinone Suppresses Cellular Proliferation, Inhibits VEGF Production and Obstructs Tumor Progression and Invasion in the Rat Model of DMH-Induced Colon Carcinogenesis," Pharmacology \& Pharmacy, Vol. 4, No. 1, 2013, pp. 7-17. doi:10.4236/pp.2013.41002

[46] G. M. Ganea, S. O. Fakayode and J. N. Losso, et al., "Delivery of Phytochemical Thymoquinone Using Molecular Micelle Modified Poly(D,L lactide-co-glycolide) (PLGA) Nanoparticles," Nanotechnology, Vol. 21, No. 28, 2010, Article ID: 285104. doi:10.1088/0957-4484/21/28/285104

[47] A. M. Ahmed, E. M. Al-Olayan, M. A. M. Aboul-Soud and A. A. Al-Khedhairy, "The Immune Enhancer, Thymoquinone, and the Hope of Utilizing the Immune System of Aedes Caspius against Disease Agents," African Journal of Biotechnology, Vol. 9, No. 21, 2010, pp. 31833195.

[48] I. O. Farah and R. A. Begum, "Effect of Nigella sativa ( $N$. sativa L.) and Oxidative Stress on the Survival Pattern of MCF-7 Breast Cancer Cells," Biomedical Sciences Instrumentation, Vol. 39, No. 1, 2003, pp. 359-364.

[49] M. A. El-Aziz, H. A. Hassan and M. H. Mohamed, et al., "The Biochemical and Morphological Alterations Following Administration of Melatonin, Retinoic Acid and Nigella sativa in Mammary Carcinoma: An Animal Model," International Journal of Experimental Pathology, Vol. 86, No. 6, 2005, pp. 383-396. doi:10.1111/j.0959-9673.2005.00448.x

[50] S. R. Samarakoon, P. B. Thabrew, D. Galhena, De-Silva and K. H. Tennekoon, "A Comparison of the Cytotoxic Potential of Standardized Aqueous and Ethanolic Extracts of a Polyherbal Mixture Comprised of Nigella sativa (Seeds), Hemidesmus Indicus (Roots) and Smilax Glabra (Rhizome)," Pharmacognosy Research, Vol. 2, No. 6, 2010, pp. 335-342. doi:10.4103/0974-8490.75451

[51] S. Rooney and M. F. Ryan, "Modes of Action of Alpha-Hederin and Thymoquinone, Active Constituents of Nigella sativa, against HEP-2 Cancer Cells," Anticancer Research, Vol. 25, No. 6B, 2005, pp. 4255-4259.

H\&E: Hematoxylin and Eosin

HPLC: High Performance Liquid Chromatography ANOVA: ANalysis Of VAriance i.p.: Initiation Phase. p.i.p.: Post Initiation Phase DMBA: Dimethylbenz(a)anthracene 